\title{
Infrared observations of NGC 3603
}

\section{The enigmatic, highly reddened sources of IRS 9*}

\author{
D. E. A. Nürnberger ${ }^{\star \star}$ \\ European Southern Observatory, Casilla 19001, Santiago 19, Chile \\ Institut für Theoretische Physik und Astrophysik, Univ. Würzburg, Am Hubland, 97074 Würzburg, Germany
}

Received 30 September 2002 / Accepted 18 March 2003

\begin{abstract}
In the framework of a multi-wavelengths study of NGC 3603 we have performed near and mid infrared imaging to investigate the nature and the evolutionary status of the highly reddened sources NGC 3603 IRS 9A-C. These sources are located on the OB cluster facing side of the massive molecular cloud core NGC 3603 MM 2 and were apparently only recently revealed from most of their natal environment by strong stellar winds and energetic radiation originating from the nearby high mass cluster stars.

On the basis of the steeply rising spectral energy distributions and the large near and mid infrared excess emission we conclude that IRS 9A-C represent (the brightest members of) a sparse association of high mass protostars, solely embedded in the gravitationally bound material of their circumstellar envelopes but largely blown free from gas + dust of the pristine molecular cloud core.

Fitting blackbody functions to the overall spectral energy distribution at near and mid infrared wavelengths we identify two dust components at temperatures of $250 \mathrm{~K}$ and $1150 \mathrm{~K}$ for IRS 9A and $250 \mathrm{~K}$ and $700-750 \mathrm{~K}$ for IRS 9B/9C. From the mid infrared fluxes we deduce lower limits for the gas + dust masses of the circumstellar envelopes: $\gtrsim 0.1 \mathcal{M}_{\odot}$ for IRS 9A and $\gtrsim 3 \times 10^{-3} \mathcal{M}_{\odot}$ for IRS 9B/9C. Estimates of the total luminosities are on the order of $10^{5} L_{\odot}$ and $10^{3} L_{\odot}$ for IRS 9A and IRS 9B / 9C, respectively.

Finally, our interpretation of IRS 9A-C being protostars is supported by recent ATCA data. The measured $3 \mathrm{~cm}$ and $6 \mathrm{~cm}$ radio fluxes of IRS 9A can neither be explained by non-thermal emission nor by thermal dust emission. From the spectral in$\operatorname{dex} \alpha_{\text {radio }} \sim 0.3$ we conclude that the observed radio emission is thermal bremsstrahlung and most likely excited externally by ionizing photons originating from the nearby $\mathrm{OB}$ cluster.
\end{abstract}

Key words. stars: early-type - stars: formation - stars: pre-main sequence - stars: individual: NGC 3603 IRS 9 stars: individual: NGC 3603 IRS 9A-C - ISM: individual objects: NGC 3603

\section{Introduction}

Molecular line and dust continuum observations (e.g. Chini et al. 1991; Bronfman et al. 1996) have shown that young OB stars are usually deeply embedded in and obscured by massive environments of gas and dust. They contract to main sequence, hydrogen burning temperatures and densities on time scales which are much shorter than typical accretion time scales (Yorke 1986). Thus, in contrast to low mass stars, young stellar objects of higher mass $\left(\mathcal{M}>8 \mathcal{M}_{\odot}\right)$ are in general not detectable at optical wavelengths throughout their (short) premain sequence phase.

Because they emit a large amount of hydrogen-ionizing Lyman-Continuum photons young OB stars ionize their circumstellar material resulting in the formation of ultracompact

\footnotetext{
^ Based on observations performed at the Las Campanas Observatory of the Carnegie Institution and at the European Southern Observatories on La Silla and Paranal, Chile.

$\star \star$ e-mail: dnuernbe@eso.org
}

H II regions (UCHIIs; e.g. Hollenbach et al. 1994; Yorke \& Welz 1996). They appear as compact, luminous infrared (IR) sources - similar to the Becklin-Neugebauer object in Orion - and their spectral energy distributions (SEDs) show a characteristic steep increase with IR wavelengths. For evolutionary stages prior to the UCH II phase, the massive parental cloud cores even cause near/mid IR radiation to be absorbed and reradiated at longer (mid IR, far IR and sub-mm) wavelengths.

Consequently, high mass protostars are rare, difficult to find and difficult to study. This fact is directly reflected by the so far known sample of confirmed class 0 protostars, where just 4 sources out of 42 may evolve into high mass main sequence stars (W3OH-TW, Trifid-TC3, G 34.24+0.13 MM, IRAS 23385; see Table 1 of André et al. 2000 and the references therein). Some few, further protostellar candidates, e.g. G 034.402+00.233 (Bronfman et al. 2001), will need more detailed characterization and/or confirmation of their evolutionary status. 

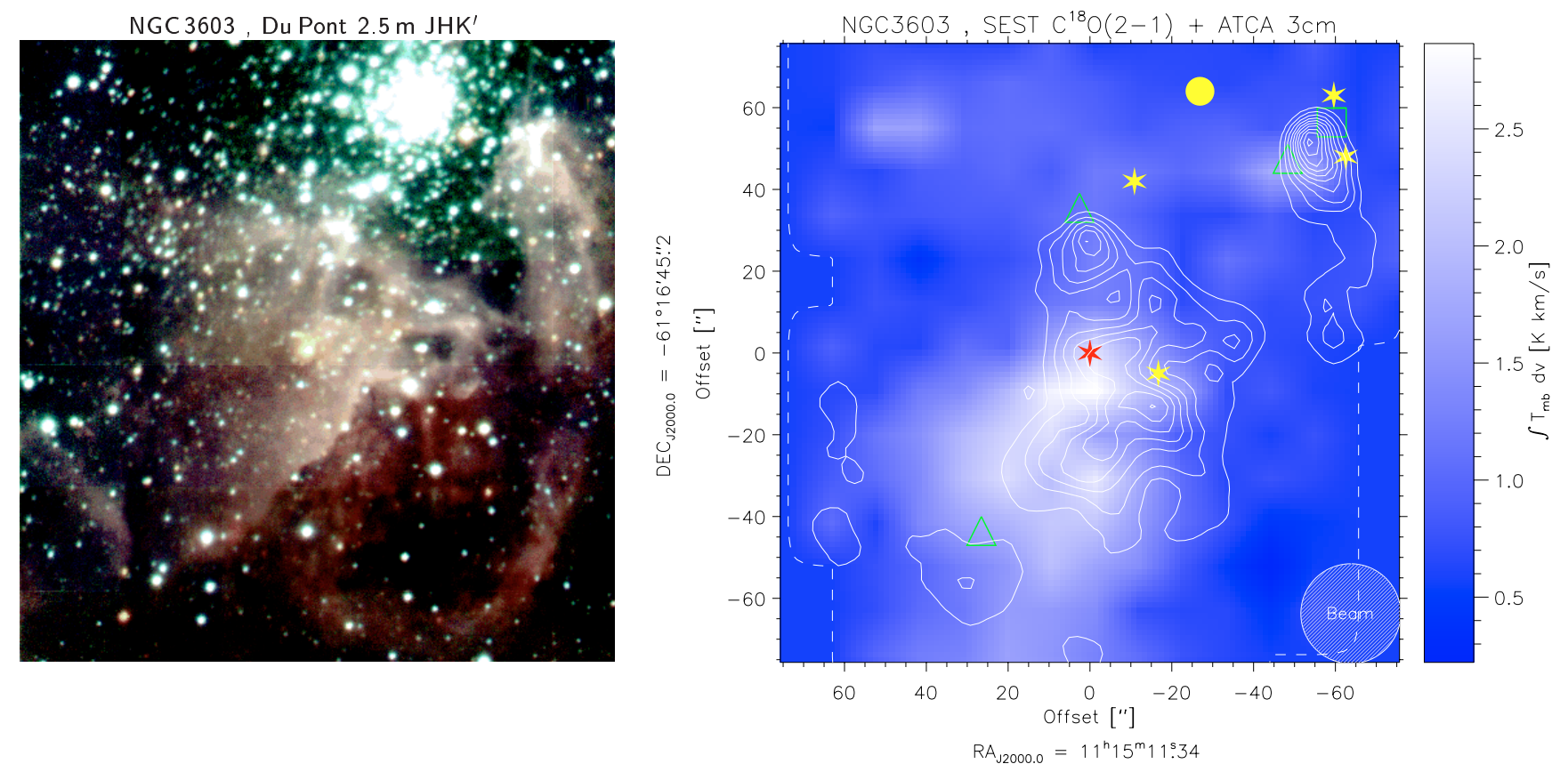

Fig. 1. 3-colour composite $J H K^{\prime}$ image (left) as well as $\operatorname{SEST~} \mathrm{C}^{18} \mathrm{O}(2-1)$ and ATCA $3.4 \mathrm{~cm}$ data (right). In both panels the field of view is $150^{\prime \prime} \times 150^{\prime \prime}$ centered on NGC 3603 IRS 9. North is up, East is left. In the right frame the $3.4 \mathrm{~cm}$ emission originating from ionized material is displayed in contour lines on top of the $\mathrm{C}^{18} \mathrm{O}(2-1)$ emission from cold molecular gas. The NGC $3603 \mathrm{OB}$ cluster is marked by the dot, selected IR sources (Frogel et al. 1977) are indicated by asterisks. Positions of OH and $\mathrm{H}_{2} \mathrm{O}$ maser (Caswell \& Haynes 1987; Caswell et al. 1989; Caswell 98) are given by squares and triangles, respectively. Additionally, the SEST beam $\left(H P B W \sim 24^{\prime \prime}\right)$ at the frequency of the $\mathrm{C}^{18} \mathrm{O}(2-1)$ line is given in the lower right corner. For comparison, the ATCA beam at $3.4 \mathrm{~cm}$ is much smaller $\left(\sim 7^{\prime \prime}\right)$. The ATCA data were obtained by De Pree et al. (1999), the SEST data are taken from Nürnberger et al. (2002).

In order to circumvent the problem of high (intrinsic) extinction we here investigate a scenario where young high mass stars form in the violent neighbourhood of a cluster of early type main sequence stars. The presence of already evolved OB type stars provides a wealth of energetic photons as well as powerful stellar winds which are capable to evaporate and disperse the surrounding interstellar medium, setting nearby young stars free at a relatively early evolutionary stage. Such premises are given in NGC 3603 which is located in the Carina spiral arm at a distance of about 7 kpc (e.g. Melnick et al. 1989; Crowther \& Dessart 1998; DePree et al. 1999; Pandey et al. 2000; Nürnberger et al. 2002).

NGC 3603 is amongst the most luminous, optically visible H II regions in our Galaxy, being powered by a massive cluster of OB stars (Goss \& Radhakrishnan 1969) which shows the highest density of high mass stars known in the Galaxy (Melnick et al. 1989; Moffat et al. 1994; Drissen et al. 1995; Hofmann et al. 1995; see Fig. 1, left panel). The hot cluster stars have a significant impact on the surrounding gas and dust, on the one hand by providing a hugh amount of ionizing photons (Lyman continuum flux $\sim 10^{51} \mathrm{~s}^{-1}$; Kennicutt 1984; Drissen etal. 1995) and on the other hand by compressing and/or dispersing adjacent molecular clumps through fast stellar winds (speeds up to several $100 \mathrm{~km} \mathrm{~s}^{-1}$; Balick et al. 1980).

One of these molecular clumps (NGC3603 MM 2; Nürnberger et al. 2002) is located about 1.'3 towards the south of the NGC 3603 OB cluster (see Fig. 1, right panel). On the cluster facing side of this clump one finds the target of our present study, the highly reddened sources of NGC 3603 IRS 9.

\section{Observations and data reduction}

The infrared data included in this work were obtained at the La Silla and Paranal observatories of the European Southern Observatory (ESO) as well as at Las Campanas Observatory of the Carnegie Institution of Washington (CIW) between May 1996 and December 2001. The most relevant parameters of these observations are summarized in Table 1.

At all wavelengths we made use of a dithering (sometimes also named jitter) technique with typical offsets in the range of $20-30^{\prime \prime}$. For the acquisition of the $L-, M-, N$ - and $Q$-band data we additionally applied chopping and nodding methods with typical throws of the order 5-20".

For each data set, all basic steps of data reduction were performed using standard routines within the IRAF and IDL software packages. Exhaustive descriptions of the succeeding processing can be found in Nürnberger \& Petr-Gotzens (2002) for the near IR data and in Nürnberger \& Stanke (2003) for the mid IR data.

\section{Astrometric and photometric calibration}

The target position is given by the source NGC 3603 IRS 9A $\left(\mathrm{RA}_{\mathrm{J} 2000}=11^{\mathrm{h}} 15^{\mathrm{m}} 11^{\mathrm{s}} .34 \pm 0.05, \mathrm{Dec}_{\mathrm{J} 2000}=-61^{\circ} 16^{\prime} 45^{\prime \prime} \cdot 2 \pm 00^{\prime} 6\right.$; ICRS frame) which is located about 1.2 towards the south of the center of the OB cluster. These coordinates of IRS 9A were determined on our deep VLT + ISAAC $K_{\mathrm{s}}$-band mosaic (Nürnberger \& Petr-Gotzens 2002) relative to the sources 8959-1919-1, 8959-1813-1 and 8959-1245-1 of the TYCHO-2 
Table 1. Overview on the obtained data sets.

\begin{tabular}{ccccccccc}
\hline \hline Instrument & Date & $\begin{array}{c}\text { Seeing } \\
{\left[{ }^{\prime \prime}\right]}\end{array}$ & $\begin{array}{c}\text { Plate scale } \\
{\left[{ }^{\prime \prime} / \mathrm{pixel}\right]}\end{array}$ & Filter & $\begin{array}{c}\lambda_{\mathrm{c}} \\
{[\mu \mathrm{m}]}\end{array}$ & $\begin{array}{c}\Delta \lambda \\
{[\mu \mathrm{m}]}\end{array}$ & $\begin{array}{c}\text { Exp. time } \\
{[\mathrm{s}]}\end{array}$ & $\begin{array}{c}\text { Frames } \\
\text { on-source }\end{array}$ \\
\hline LCO 2.5 m \& NIRCAM & $25 .-26.05 .96$ & $0.7-0.9$ & 0.348 & $J^{\prime}$ & 1.25 & 0.20 & 5.0 & 20 \\
& & & & $H$ & 1.65 & 0.30 & 5.0 & 20 \\
& & & & $K^{\prime}$ & 2.16 & 0.33 & 3.0 & 34 \\
& $29 .-30.05 .99$ & $0.6-0.8$ & 0.348 & $J^{\prime}$ & 1.25 & 0.20 & 3.0 & 10 \\
& & & & $H$ & 1.65 & 0.30 & 3.0 & 10 \\
\hline VLT Antu \& ISAAC & $28 .-30.05 .99$ & $0.4-0.7$ & 0.147 & $K_{\mathrm{s}}$ & 2.16 & 0.27 & $60^{a}$ & 60 \\
& $25 .-26.06 .99$ & $0.4-0.5$ & 0.070 & $L$ & 3.78 & 0.58 & $2.4^{b}$ & 20 \\
& & & & $M_{\mathrm{nb}}$ & 4.66 & 0.10 & $2.4^{c}$ & 20 \\
\hline ESO 3.6 m \& TIMMI 2 & $22 .-24.01 .01$ & $\sim 0.5$ & 0.296 & $N 11.9$ & 11.59 & 0.60 & $110-400$ & 10 \\
& $28 .-31.12 .01$ & $\sim 0.5$ & 0.296 & $N 11.9$ & 11.59 & 0.60 & $110-400$ & 10 \\
& & & 0.202 & $Q 1$ & 17.75 & 0.40 & 390 & 6 \\
\hline
\end{tabular}

Notes: ${ }^{a}$ DIT $=3.000 \mathrm{~s}$, NDIT $=20 ;{ }^{b}$ DIT $=0.020 \mathrm{~s}$, NDIT $=122 ;{ }^{c}$ DIT $=0.021 \mathrm{~s}$, NDIT $=113$.

catalogue $^{1}$ (Hog et al. 2000). Due to the subsequent registering of IRS 9A on our individual data sets, the resulting position accuracy is limited by the corresponding pixel scaling which is given in Col. 4 of Table 1.

All observations were performed under photometric conditions and at airmasses between 1.2 and 1.5. For the flux calibration of the $J H K$ data we observed each night several near IR standard stars from the compilations of Casali \& Hawarden (1992) and Persson et al. (1998): FS 19, S279-F, S064-F and S791-C. For the calibration of the $L$ - and $M$-band data suitable standard stars (namely HR 4450 and HR 4520) were selected from the list of van der Bliek et al. (1996). Finally, the $N$ - and $Q$-band data were calibrated with ISO standard stars, for which Cohen (1998) provide mid IR flux estimates based on SED models: HD 4128, HD 29139, HD 32887, HD 47105, HD 81420 and HD 81797.

\section{Results and discussion}

\subsection{Near IR and mid IR images}

In Fig. 2 we present our near IR and mid IR data obtained with the Las Campanas $2.5 \mathrm{~m}$ telescope $\left(J^{\prime}, H, K^{\prime}\right)$, the VLT Antu $\left(K_{\mathrm{s}}, L, M_{\mathrm{nb}}\right)$ and the La Silla $3.6 \mathrm{~m}$ telescope $(N 11.9, Q 1)$. Each image covers a field-of-view of $18 .^{\prime \prime} 1 \times 18$.' $^{\prime} 1$, which corresponds to about $0.6 \mathrm{pc} \times 0.6 \mathrm{pc}$ at the distance of $7 \mathrm{kpc}$ (see the discussion on the distance of NGC 3603 in Nürnberger \& Petr-Gotzens 2002 and Nürnberger et al. 2002), and is centered on the brightest source of IRS 9, which - following the nomenclature indroduced by Frogel et al. (1977) - is named IRS 9A here.

In the $K_{\mathrm{s}}$ panel we have marked several $K$-band excess sources identified by Tapia et al. (2001). All of them are also (rather) bright in our $L$ - and $M_{\mathrm{nb}}$-band data. Furthermore, on our mid IR images three sources (IRS 9A, IRS 9B and IRS 9C) clearly stand out as strong $11.9 \mu \mathrm{m}$ and $18 \mu \mathrm{m}$ emitters. The strong near and mid IR excess emission indicates the presence of massive circumstellar envelopes of gas and dust.

\footnotetext{
${ }^{1}$ Accessible via the Centre de Données astronomiques de Strasbourg (CDS).
}

\subsection{Colour-magnitude and two-colour diagrams}

The presence of circumstellar material is confirmed by the near IR colour-magnitude and two-colour diagrams (hereafter appreviated with CMD and TCD, respectively) shown in Fig. 3, where we have corrected for (a minimum of) 4.5 of visual foreground extinction (see Moffat 1983 and Melnick et al. 1989) by using the extinction transformations given by Rieke \& Lebofsky (1985). In both diagrams the loci of IRS 9A-C are compared to those of stars located in the central $12^{\prime \prime} \times 12^{\prime \prime}$ of the NGC 3603 OB cluster (Eisenhauer et al. 1998).

Unambiguously, the near IR colours of IRS 9A-C are clearly offset from those of (high mass) main sequence stars. From the $(J-K)-M_{J}$ diagram we derive reddening vectors of $A_{V} \sim 22^{\mathrm{m}}$ for IRS 9A, $\sim 10^{\mathrm{m}}$ for IRS $9 \mathrm{~B}$ and $\sim 25^{\mathrm{m}}$ for IRS 9C. If we follow these reddening vectors back to the main sequence they would imply very high stellar masses in the range of $30 \mathcal{M}_{\odot}$ to $100 \mathcal{M}_{\odot}$. Taking into account the location of NGC 3603 at the outer edge of the Carina spiral arm, i.e. the bulk of field stars lies in front of the cluster (Sagar et al. 2001), we consider it unlikely that IRS 9A-C represent background stars reddened by such high values. This conclusion is supported by the fact that IRS 9A-C are located very close to each other on the sky but show IR colours significantly different from each other.

According to Strom et al. (1993) low mass stars possessing near IR excess emission usually populate three distinct regions of the $(H-K)-(J-H)$ diagram: sources associated with low excess emission (so-called weak-line T Tauri stars; WTTS) are found in zone I, while zones II and III feature sources with high excess emission caused by circumstellar disks (classical T Tauri stars; CTTS) and surrounding envelopes (protostars), respectively. The same or at least a similar scenario should hold for intermediate and high mass stars. Therefore, the strong reddening of IRS $9 \mathrm{~A}-\mathrm{C}$ might be convincingly explained by intrinsic extinction due to the presence of large amounts of circumstellar gas and dust.

Moreover, IRS 9A-C appear much younger and more massive than NGC 3603's intermediate and low mass pre-main sequence stars, which are characterized best by isochrones in 

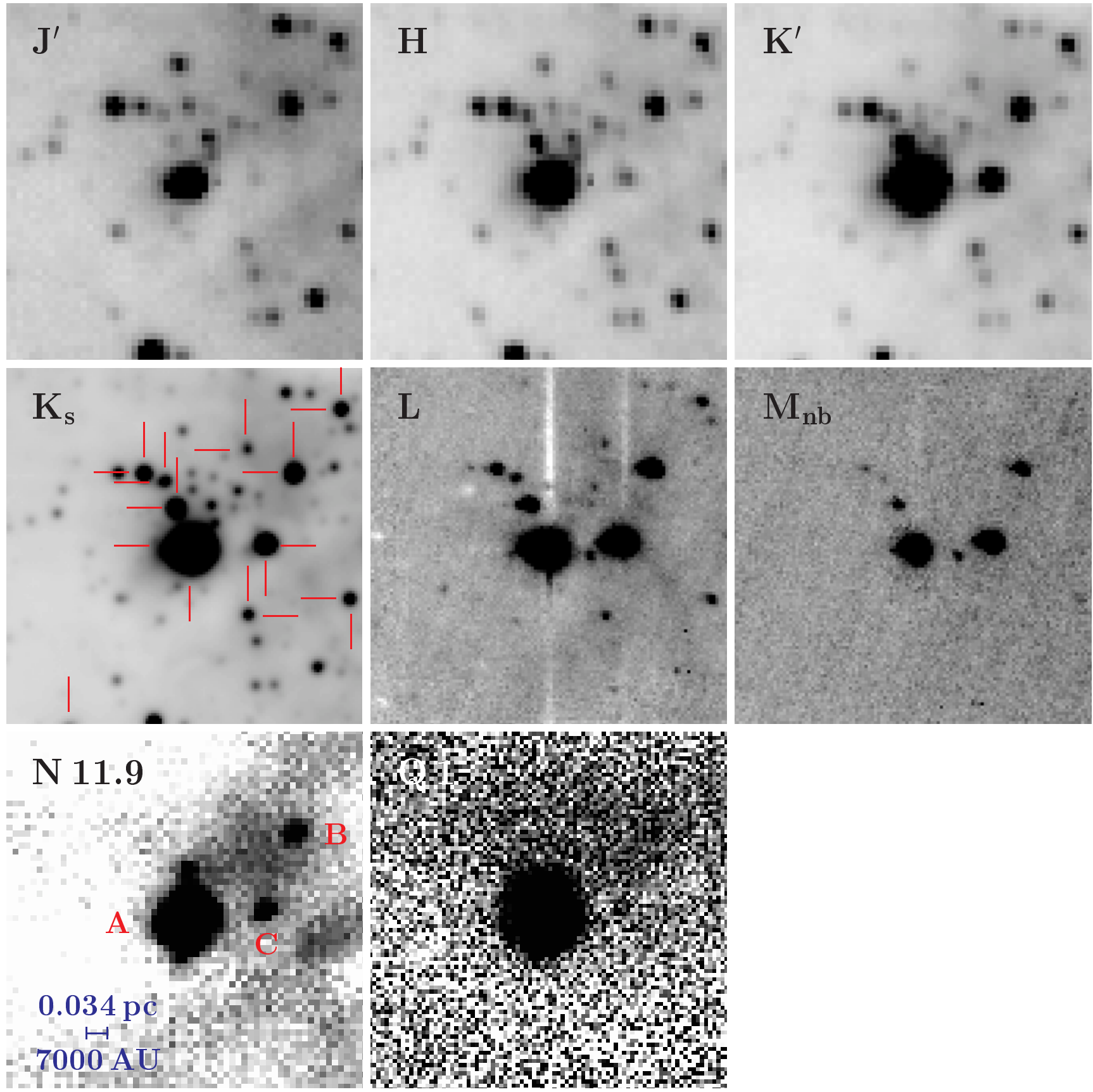

Fig. 2. Near IR and mid IR data obtained with the Las Campanas $2.5 \mathrm{~m}$ telescope (top row), the VLT Antu (middle row) and the La Silla $3.6 \mathrm{~m}$ telescope (bottom row). Each image is centered on IRS 9A and covers an area of $18^{\prime} .1 \times 18^{\prime \prime}$. 1 , which corresponds to $0.6 \mathrm{pc} \times 0.6 \mathrm{pc}$ at the distance of $7 \mathrm{kpc}$. In the $K_{\mathrm{s}}$ image markers indicate sources with significant $K$ band excess as identified by Tapia et al. (2001). The highly reddened sources NGC 3603 IRS 9A-C are annotated in the $N$ band image.

Table 2. Measured apparent magnitudes (without applying any correction for foreground extinction).

\begin{tabular}{ccccccccc}
\hline \hline Source & $J^{\prime}$ & $H$ & $K^{\prime}$ & $\begin{array}{c}K_{\mathrm{s}} \\
{[\mathrm{mag}]}\end{array}$ & $\begin{array}{c}L \\
{[\mathrm{mag}]}\end{array}$ & $\begin{array}{c}M_{\mathrm{nb}} \\
{[\mathrm{mag}]}\end{array}$ & $\begin{array}{c}N 11.9 \\
{[\mathrm{mag}]}\end{array}$ & $\begin{array}{c}Q 1 \\
{[\mathrm{mag}]}\end{array}$ \\
\hline IRS 9A & $12.87 \pm 0.20$ & $10.92 \pm 0.20$ & $8.55 \pm 0.20$ & saturated & $6.00 \pm 0.02$ & $5.53 \pm 0.02$ & $0.54 \pm 0.03$ & $-1.85 \pm 0.03$ \\
IRS 9B & $14.63 \pm 0.20$ & $13.94 \pm 0.20$ & $11.96 \pm 0.20$ & $12.23 \pm 0.02$ & $8.75 \pm 0.02$ & $8.27 \pm 0.02$ & $4.02 \pm 0.03$ & $1.94 \pm 0.03$ \\
IRS 9C & $(16.71 \pm 0.20)$ & $15.34 \pm 0.20$ & $11.58 \pm 0.20$ & $11.85 \pm 0.02$ & $7.01 \pm 0.02$ & $6.35 \pm 0.02$ & $4.03 \pm 0.03$ & $2.21 \pm 0.03$ \\
\hline
\end{tabular}



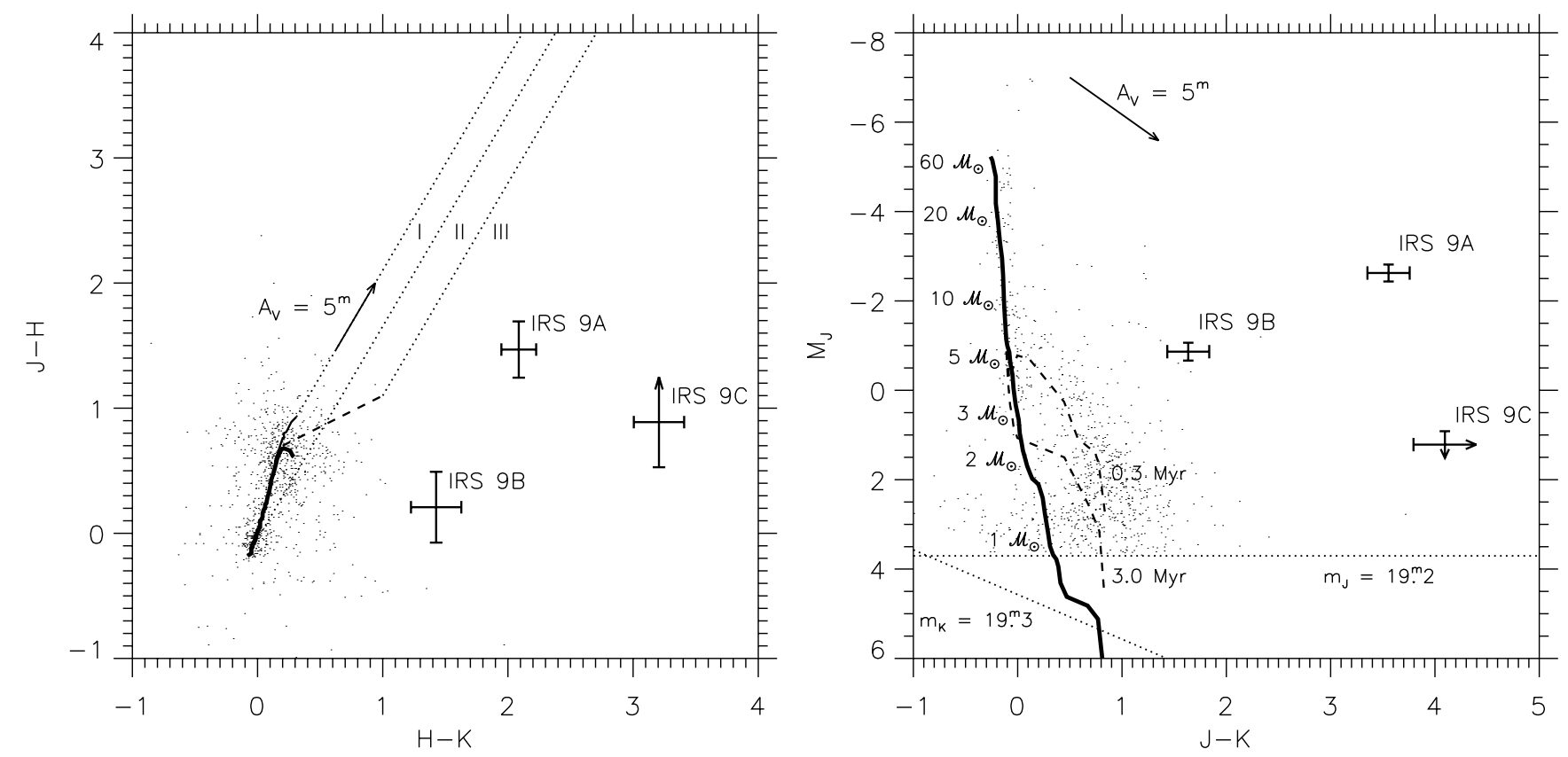

Fig. 3. Near IR colour-magnitude and two-colour diagrams of the central $12^{\prime \prime} \times 12^{\prime \prime}$ of the NGC 3603 OB cluster (Eisenhauer et al. 1998) with the corresponding positions of IRS 9A, IRS 9B and IRS 9C indicated. Both diagrams impressively demonstrate the extreme youth of IRS 9A-C in comparison to the population of the OB cluster, in particular, as we already have corrected for the foreground extinction of $A_{V}=4 \mathrm{~m} .5$.

the range 0.3-3.0 Myr (following Eisenhauer et al. 1998 and Brandl et al. 1999). In fact, the loci of IRS 9A-C in our near IR CMD and TCD suggest quite early evolutionary stages (probably ages $\leq 10^{5} \mathrm{yr}$ ), consistent with that of fairly massive $\left(\mathcal{M}>10 \mathcal{M}_{\odot}\right)$ protostars. However, we must keep in mind that near IR colours alone only allow an almost unambiguous distinction of sources with/without circumstellar material, but mid IR data are essential to identify protostars with excess emission due to their surrounding envelopes (Haisch et al. 2001).

Therefore, in Fig. 4 we show the positions of IRS 9A-C in $(K-N)-(J-K)$ and $(K-N)-M_{K}$ diagrams, together with some selected NGC 3603 cluster sources from the mid IR survey of Nürnberger \& Stanke (2003). The corresponding loci of main sequence stars, giants and supergiants are represented by thick, moderately thick and thin straight lines, following Koornneef (1983a, 1983b) and Ducati et al. (2001). As in Fig. 3, both diagrams of Fig. 4 are corrected for $A_{V}=4.5$, i.e. the foreground extinction measured along the line-of-sight towards the OB cluster. Again, considering the reddening vectors for interstellar extinction, it appears very unlikely that sources with extremely red colours (e.g. IRS 9A-C, 5A-C) and well separated from the main sequence are highly reddened, high mass background sources. In the case of IRS 9A-C we will provide evidence for their protostellar nature in the following sections. For a detailed discussion of the other sources (in particular, of the sources 4 and $6 \mathrm{~A}-6 \mathrm{G}$ ) we refer to Nürnberger \& Stanke (2003).

\subsection{Spectral energy distributions}

In Fig. 5 we present the SEDs of IRS 9A (top), IRS 9B (middle) and IRS 9C (bottom) as they emerge from our IR data. In addition, the results of Frogel et al. (1977), Persi et al. (1985),
Roth et al. (1987) and Tapia et al. (2001) are displayed for the purpose of comparison.

Significant discrepancies between our fluxes and those published in the literature are mostly due to the lower angular resolution and the lower sensitivity of the earlier data sets. E.g., Persi et al. (1985) and Roth et al. (1987) used quite large apertures of 7.'5 and 5.'0, respectively, for their near IR photometry. Consequently, their fluxes include contributions from neighbouring point sources and diffuse emission for which they did not correct (M. Tapia, priv. comm.). In a similar way, the higher near IR fluxes obtained by Tapia et al. (2001) for IRS 9B and 9C (although corrected for contributions of nearby point sources in the 5'. 0 aperture) might be explained by insufficient subtraction of the surrounding diffuse emission. The large aperture of 14." 5 applied by Frogel et al. (1977) on their $N$ and $Q$ band data did neither allow to resolve IRS 9A-C, nor to correct for diffuse emission properly.

In the left panels the fluxes of the three sources are only dereddened for a foreground extinction of $A_{V}=4.5$, while in the center and right panels additional intrinsic extinctions of $A_{V} \sim 10 \mathrm{~m} .5$ and $\sim 15.5$ (IRS $9 \mathrm{~A}$ ) as well as $\sim 3 . \mathrm{m} 0$ and $\sim 5.5$ (IRS 9B and IRS 9C) are taken into account. In each case the overall SED is fitted best by a combination of three Planck functions at temperatures of $22000 \mathrm{~K}, 1000-1150 \mathrm{~K}, 250 \mathrm{~K}$ (IRS 9A) and $20000 \mathrm{~K}, 650-750 \mathrm{~K}, 250-270 \mathrm{~K}$ (IRS 9B and IRS 9C), respectively. In good agreement with IRS 9A's and IRS 9B's loci in the near IR CMD and TCD, a (lower) value of $22000 \mathrm{~K}$ and $20000 \mathrm{~K}$ for the "stellar" temperature qualifies the corresponding central source as a high mass (proto-) star of spectral type B2 or earlier.

For IRS 9C we can not derive any explicit constraint on the temperature of its central source, because the SED can be successfully modeled without incorporating a stellar blackbody 


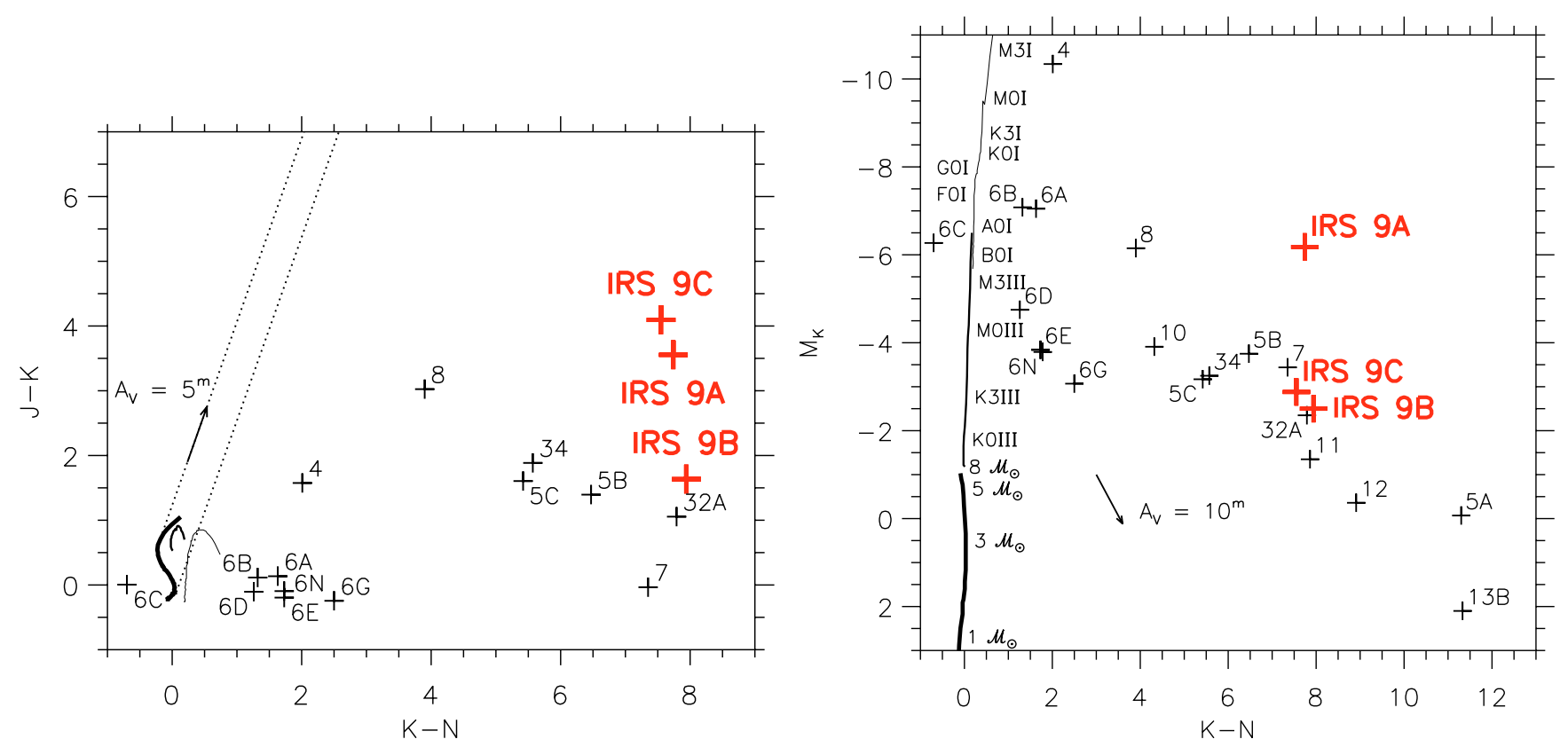

Fig. 4. Near/mid IR colour-magnitude and two-colour diagrams of some selected cluster sources from the recent mid IR survey of NGC 3603 (see Nürnberger \& Stanke 2003 for more details), with the corresponding positions of IRS 9A-C highlighted. As in Fig. 3, both diagrams are corrected for the foreground extinction of $A_{V}=4.5$. The straight lines (from thick to thin) indicate the loci of main sequence stars, giants and supergiants (Koornneef 1983a, 1983b; Ducati et al. 2001).

function at a given temperature. However, we may speculate that IRS 9C is in a similar evolutionary stage as IRS 9A and IRS 9B, consistent with its position in the near IR CMD and TCD relative to the other two sources (see Figs. 3 and 4). As one can deduce from the $(K-N)-M_{K}$ diagram (Fig. 4, right panel), the central source of IRS 9C appears to have about the same luminosity as IRS 9B. Hence, the stellar masses of both sources may be similar although IRS 9C is only marginally detected in the $J$-band, due to a somewhat higher intrinsic extinction (possibly caused by the circumstellar disk seen almost edge-on for IRS 9C, while that of IRS 9B might be face-on).

\subsection{Spectral indices $\alpha_{2.2-10 \mu m}$}

In the case of low mass pre-main sequence stars a reliable evolutionary classification scheme was originally introduced by Lada (1987), and subsequently revised and extended by André \& Montmerle (1994). It is based on the IR excess of a young source, as measured by its spectral index $\alpha_{\mathrm{IR}}=\mathrm{d} \log \left(\lambda \mathrm{F}_{\lambda}\right) / \mathrm{d} \log \lambda$ between $\lambda=2.2$ and $10 \mu \mathrm{m}$. Because the excess is attributed to the presence of circumstellar gas and dust, this IR classification predicts a progressive decrease of the amount of circumstellar material from IR class 0 to IR class III, i.e. a decrease with increasing stellar age.

For deeply embedded sources (protostars; IR class 0/I; $\alpha_{\mathrm{IR}}>0$ ) most of the circumstellar matter is distributed in an extended infalling envelope with a typical size of about $10^{4}$ AU (e.g. Adams et al. 1987; Terebey et al. 1993). For the later stages of T Tauri stars (IR class II / III; $\alpha_{\text {IR }}<0$ ) the IR emission mostly arises from circumstellar disks on the order of $100 \mathrm{AU}$ in size (e.g. Adams et al. 1988; Bertout et al. 1988; André \& Montmerle 1994). Despite of some ambiguity
- depending on the orientation of its circumstellar accretion disk an IR class II/III source could mimic the spectral characteristics of a IR class I source (see e.g. Yorke et al. 1995; Sonnhalter et al. 1995) - this classification scheme works fine and is well established for low mass sources.

Let us assume that - apart from the shorter time scales the same classification scheme holds for intermediate and high mass stars. Considering a foreground extinction of $A_{V}=4.5$ we obtain reasonable and consistent values of $1.37,1.46$ and 1.24 for the spectral indices $\alpha_{2.2-10 \mu \mathrm{m}}$ of IRS 9A, IRS 9B and IRS 9C, respectively. Together with the nearly identical dust temperatures this suggests that IRS 9A-C have a common origin and indeed form a sparse association of young sources. Furthermore and most important, the clearly positive spectral indices indicate the protostellar nature of these sources and make them comparable to IR class I objects of intermediate to high mass, e.g. identified in M 17 by Nielbock et al. (2001).

\subsection{Circumstellar gas and dust masses}

With information on the dust temperature at hand (Fig. 5) and assuming - in a first approximation - that the mid IR emission is optically thin, our measurements can be used to calculate lower limits of the hot dust masses (Natta \& Panagia 1976; Hildebrand 1983; Kraemer et al. 1999):

$\mathcal{M}_{\text {dust }}=\frac{F_{v}}{B_{v}\left(\mathrm{~T}_{\text {dust }}\right)} \cdot D^{2} \cdot \frac{4 a \rho}{3 Q_{\mathrm{e}}}$,

where $a \sim 0.01 \mu \mathrm{m}, \rho \sim 2 \mathrm{~g} \mathrm{~cm}^{-3}$ and $Q_{\mathrm{e}} \sim 10^{-4}$ (following Becklin et al. 1976) are typical parameters for the size, mass density and emissivity of dust grains in circumstellar environments. Furthermore, the Planck function $B_{v}\left(T_{\text {dust }}\right)$ 

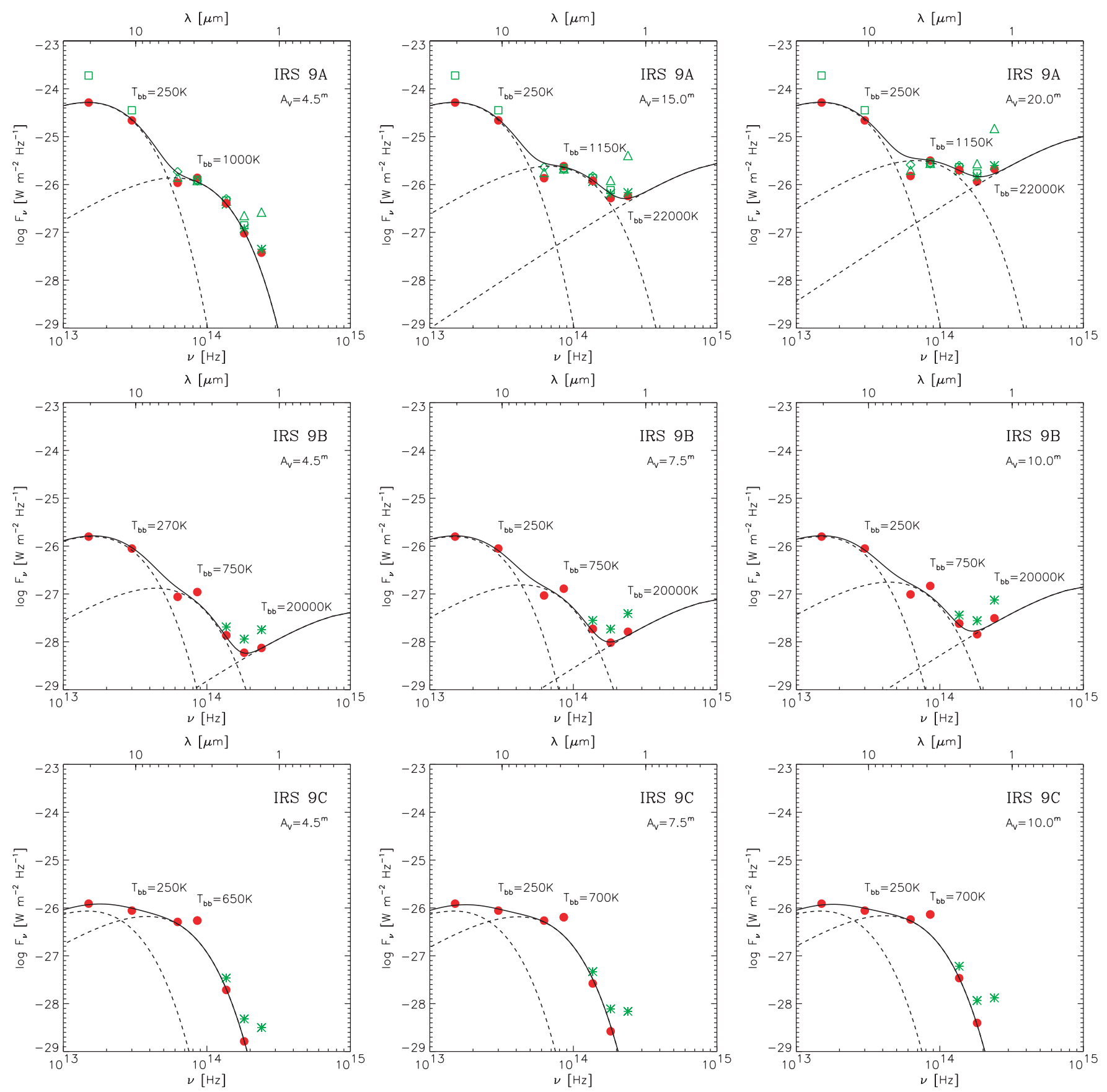

Fig. 5. Spectral energy distributions of IRS $9 A-C$ after dereddening by 4.5 of foreground extinction (left) as towards the center of the OB cluster and additionally by $10^{\mathrm{m}} .5$ and $15^{\mathrm{m}} .5$ (IRS 9A) and 3.0 and 5.5 (IRS 9B+C), respectively, of intrinsic extinction (right). The SEDs can be reproduced by a combination of three Planck functions at temperatures of about $250 \mathrm{~K}, 1100 \mathrm{~K} / 700 \mathrm{~K}$ and $22000 \mathrm{~K} / 20000 \mathrm{~K}$. The data points are taken from Frogel et al. $(1977)=\square$, Persi et al. $(1985)=\Delta$, Roth et al. $(1987)=\diamond$, Tapia et al. $(2001)=*$ and this work $=\bullet$.

gives the predicted emission by dust grains at the temperature $T_{\text {dust }} \sim 250 \mathrm{~K}$, which is taken from the colder blackbody fits to the SEDs of IRS 9A-C (see Fig. 5). For $F_{v}$ we adopt our $18 \mu \mathrm{m}$ fluxes as they determine the $250 \mathrm{~K}$ component of the SEDs. For the distance of NGC 3603 we assume $D=7 \mathrm{kpc}$.

With respect to the dust properties given above it is important to keep in mind that they may easily vary by factors of the order 2-10, depending on the actual chemical composition, structure and size distribution of the grains. For a detailed discussion of dust grains in protostellar environments we refer to the comprehensive studies by Preibisch et al. (1993, 1995), Krügel \& Siebenmorgen (1994), Ossenkopf \& Henning (1994), Suttner et al. (1999) and Suttner \& Yorke (2001), which revealed fundamental differences of grain properties in comparison to those of the interstellar dust (see e.g. Mathis et al. 1977; Draine \& Lee 1984; Draine \& Li 2001; Li \& Draine 2001; Weingartner \& Draine 2001).

In Table 3 we list our estimates for the hot dust masses of IRS 9A-C, together with the coordinates, mid IR fluxes and dust temperatures. The latter are derived from the SEDs 
Table 3. Coordinates, mid IR fluxes and dust properties.

\begin{tabular}{cccccrrrc}
\hline \hline Source & $\begin{array}{c}\mathrm{RA}_{K \mathrm{~s}}^{a} \\
(\mathrm{~J} 2000)\end{array}$ & $\begin{array}{c}\operatorname{Dec}_{K \mathrm{~s}}^{a} \\
\left(\mathrm{~J}_{2000}\right)\end{array}$ & $\begin{array}{c}\mathrm{RA}_{N 11.9}^{b} \\
(\mathrm{~J} 2000)\end{array}$ & $\begin{array}{c}\operatorname{Dec}_{N 11.9}^{b} \\
(\mathrm{~J} 2000)\end{array}$ & $\begin{array}{c}F_{11.9 \mu \mathrm{m}} \\
{[\mathrm{Jy}]}\end{array}$ & $\begin{array}{c}F_{18 \mu \mathrm{m}} \\
{[\mathrm{Jy}]}\end{array}$ & $\begin{array}{c}T_{\text {dust }} \\
{[\mathrm{K}]}\end{array}$ & $\begin{array}{c}\mathcal{M}_{\text {dust }} \\
{\left[\mathcal{M}_{\odot}\right]}\end{array}$ \\
\hline IRS 9A & $11: 15: 11.34$ & $-61: 16: 45.2$ & $11: 15: 11.34$ & $-61: 16: 45.2$ & 21.88 & 51.69 & 250 & $>1.1 \times 10^{-3}$ \\
IRS 9B & $11: 15: 10.59$ & $-61: 16: 41.0$ & $11: 15: 10.62$ & $-61: 16: 41.1$ & 0.89 & 1.58 & 250 & $>3.4 \times 10^{-5}$ \\
IRS 9C & $11: 15: 10.80$ & $-61: 16: 44.8$ & $11: 15: 10.86$ & $-61: 16: 44.9$ & 0.88 & 1.23 & 250 & $>2.6 \times 10^{-5}$ \\
\hline
\end{tabular}

Notes: ${ }^{a}$ Nürnberger \& Petr-Gotzens (2002); ${ }^{b}$ Nürnberger \& Stanke (2003).

presented in Fig. 5. Using the standard gas-to-dust ratio of 100:1 (e.g. Whittet 1992) these values correspond to circumstellar gas + dust masses of about $0.1 \mathcal{M}_{\odot}$ for IRS 9A and $3 \times 10^{-3} \mathcal{M}_{\odot}$ for IRS 9B / 9 C.

Independent of whether or not the observed mid IR emission is optically thin, above mass estimates represent strict lower limits to the actual circumstellar gas + dust masses because our SED models lack important information on far IR and (sub-) millimeter fluxes. As noted by Kraemer et al. (1999), far IR and (sub-) millimeter emission usually arises from larger and cooler grains which would contribute - if present - at least one order of magnitude more mass (see Gezari et al. 1985). Dust continuum emission at (sub-) millimeter wavelengths predominantly originates from the outer, cold parts of circumstellar environments (disks and/or envelopes) and is usually optically thin. Thus, mass estimates derived from (sub-) millimeter data not only represent an important complement to gas + dust masses derived from IR data, but they are generally more reliable.

Assuming the presence of an envelope of radius $\mathcal{R}_{\text {env }}<$ $7000 \mathrm{AU}$ (see the $N 11.9$ and $Q 1$ panels of Fig. 2) around IRS 9A and a homogeneous distribution of the circumstellar material within this envelope, we can derive (lower limit) averages for the volume and column densities: $n\left(\mathrm{H}_{2}\right) \gtrsim 1.3 \times$ $10^{4} \mathrm{~cm}^{-3}$ and $\mathcal{N}\left(\mathrm{H}_{2}\right) \gtrsim 1.4 \times 10^{21} \mathrm{~cm}^{-2}$, respectively. We note that - if we assume an intrinsic extinction of $10-15^{\mathrm{m}}$, as derived via the dereddening of IRS 9A's SED - a column density of $\mathcal{N}\left(\mathrm{H}_{2}\right)=0.94 \times 10^{21} \cdot A_{V}=(9.4-14.1) \times 10^{21} \mathrm{~cm}^{-2}$ (Bohlin et al. 1978; Dickman 1978) would be required, which would correspond to a circumstellar gas + dust mass of $\sim 0.7-1.1 \mathcal{M}_{\odot}$. As already discussed above, this can easily be the case if colder dust components (primarily contributing to the SED at far IR to millimeter wavelengths) are indeed missed so far.

Recent observations with the Australia Telescope Compact Array (ATCA) yielded for NGC 3603 IRS 9A radio fluxes of $F_{3 \mathrm{~cm}}=19.0 \pm 0.2 \mathrm{mJy}$ and $F_{6 \mathrm{~cm}}=15.2 \pm 0.5 \mathrm{mJy}$ (A. Reimer, nee Mücke, priv. comm.; see also Fig. 1 of Mücke et al. 2002). The corresponding spectral index $\alpha_{\text {radio }} \sim 0.3$ clearly excludes significant contributions from non-thermal components and indicates a preferentially thermal origin of the radio emission. However, even if we take into account a dust component colder than $250 \mathrm{~K}$, the observed radio fluxes of IRS 9A are so high that they can not be explained by thermal emission arising from reasonable amounts of reasonably cold dust.

Instead, one may consider optically thin thermal bremsstrahlung dominating the radio emission. Then we can calculate the photon rate required to ionize the material around IRS 9A following the formula (see Kurtz et al. 1999; Rubin 1968)

$N_{\text {Ly }}=4.76 \times 10^{41} \cdot f^{-1} v^{0.1} T_{\mathrm{e}}^{-0.45} F_{v} D^{2}$,

where the frequency $v$ is in $\mathrm{GHz}$, the corresponding flux $F_{v}$ in Jy and the distance $D$ in kpc. The correction factor $\mathrm{f}$ depends on both frequency and electron temperature and should be set to 0.985 if one adopts $v=10 \mathrm{GHz}(\lambda=3 \mathrm{~cm})$ and $T_{\mathrm{e}}=$ $10^{4} \mathrm{~K}$ (Mezger \& Henderson 1967). Hence, we end up with $N_{\text {Ly }} \sim 10^{40} \mathrm{~s}^{-1}$ which is by far too low for IRS 9A harbouring an early type main sequence star (Vacca et al. 1996), i.e. internal ionisation can not explain the measured radio fluxes. What about external ionisation by the high mass stars of the nearby OB cluster? In fact, the central cluster in NGC 3603 provides a Lyman continuum flux of about $10^{51} \mathrm{~s}^{-1}$ (Kennicutt 1984; Drissen et al. 1995), which roughly converts into $10^{46} \mathrm{~s}^{-1}$ when taking into account the projected distance $(\sim 2.4 \mathrm{pc})$ of IRS 9A from the cluster center. This value would even leave sufficient room for dust attenuation and/or a larger linear distance between IRS 9A and the OB cluster.

\subsection{Luminosities}

If we imply that the emissivity $Q_{\mathrm{e}}$ of the dust grains at mid IR wavelengths is proportional to $\lambda^{-1}$ we can calculate the total luminosities of the sources via the equation

$L=4 D^{2} \cdot \sigma \cdot \lambda T^{5} \cdot \frac{F_{v}}{B_{v}\left(T_{\text {dust }}\right)}$,

with the constant $\sigma=1.51 \times 10^{-5} \mathrm{~J} \mathrm{~s}^{-1} \mathrm{~m}^{-3} \mathrm{~K}^{-5}$ (following Natta \& Panagia 1976 and Felli \& Stanga 1987). When setting the other parameters as in Sect. 4.5, we obtain luminosities of $L \sim 2.3 \times 10^{5} L_{\odot}, L \sim 7.0 \times 10^{3} L_{\odot}$ and $L \sim 5.5 \times 10^{3} L_{\odot}$ for IRS 9A, IRS 9B and IRS 9C, respectively.

Together with the "stellar" temperatures derived from the SED modeling (see Fig. 5) our estimates for the luminosities allow us to roughly place IRS 9A-C in the HR diagram. Comparison of their loci with pre-main sequence evolutionary tracks of Bernasconi \& Maeder (1996; Fig. 6) indicates rather high masses for our young sources, namely $\sim 40 \mathcal{M}_{\odot}$ for IRS 9A and $\sim 9-10 \mathcal{M}_{\odot}$ for IRS 9B / 9 C. Furthermore, from the corresponding contraction times given in Table 2 of Bernasconi \& Maeder (1996) we deduce an age of $\sim 10^{4} \mathrm{yr}$ for IRS 9A and $\sim 10^{5} \mathrm{yr}$ for IRS 9B / 9C, which is in good agreement with the sources' loci in the CMD and TCD (see Fig. 3). We also note 


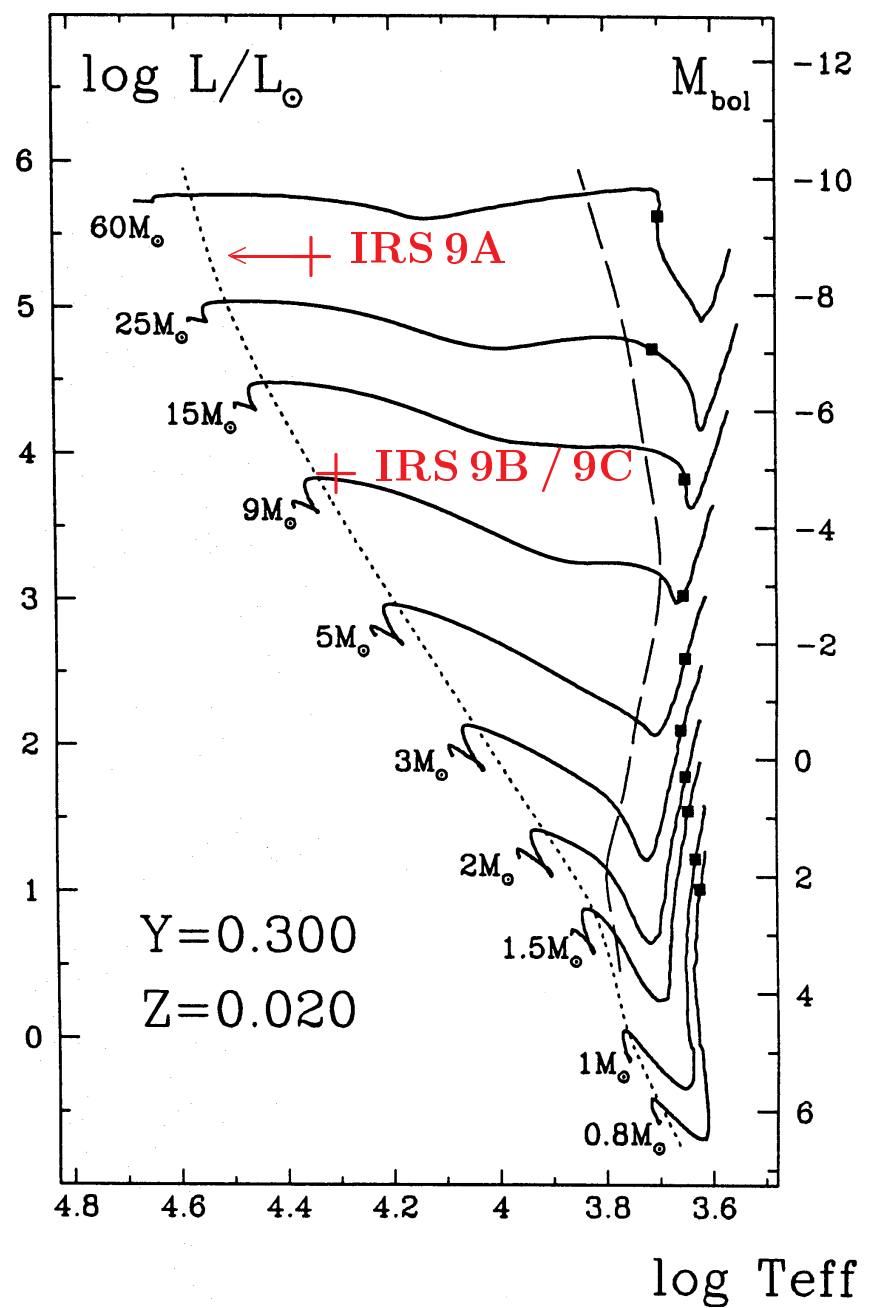

Fig. 6. Loci of IRS 9A-C in the HR diagram in comparison to premain sequence evolutionary tracks of Bernasconi \& Maeder (1996; see their Fig. 5). Following their notation filled squares mark the ignition of Deuterium burning. The long dashed line corresponds to the stage where the convective envelope disappears and the stars begin the totally radiative portion of the tracks. The short dashed line indicates the part of the tracks where the convective core first appears. The left ends of the tracks mark the zero-age main sequence.

that - if we assume IRS9A-C to be coeval $-T_{\text {eff }}$ of IRS 9A would be constraint to about $32000 \mathrm{~K}$ (as indicated by arrows in Figs. 6 and 7).

In addition, in Fig. 7 the loci of IRS 9A-C in the HR diagram are compared to evolutionary tracks of pre-main sequence stars which accrete material at a given constant rate (Yorke \& Sonnhalter 2002) as well as to pre-main sequence tracks of non-accreting stars following D’Antona \& Mazzitelli (1994) and Iben (1965). We deduce that both IRS 9B and IRS 9C may harbour a protostellar source of radius $\sim 50 \mathcal{R}_{\odot}$, accreting circumstellar material at the rate of about $10^{-3} \mathcal{M}_{\odot} \mathrm{yr}^{-1}$. In a similar way, IRS 9A can be described as a protostar of radius $\sim 1000 \mathcal{R}_{\odot}$ with a significantly higher accretion rate.

We emphasize that the uncertainties associated with both our temperature and luminosity determination exceed the size of the symbols shown in the HR diagrams of Figs. 6 and 7. However, as the pre-main sequence evolutionary tracks at

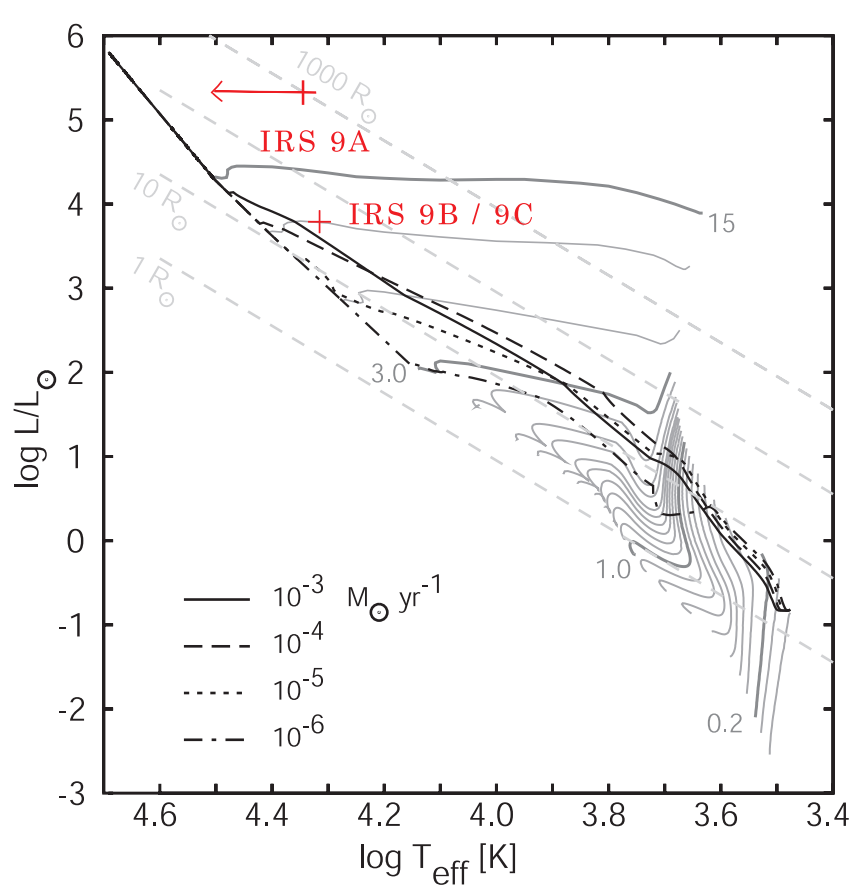

Fig. 7. Loci of IRS 9A-C in the HR diagram in comparison to evolutionary tracks of pre-main sequence stars accreting material at a constant rate (following Yorke \& Sonnhalter 2002; see their Fig. 1). For comparison, the evolutionary pre-main sequence tracks of nonaccreting stars according to D'Antona \& Mazzitelli (1994) and Iben (1965) are given as solid grey lines. Dashed grey lines indicate stellar radii.

Table 4. Estimated properties of the central sources.

\begin{tabular}{cccccc}
\hline \hline Source & $\begin{array}{c}A_{V}^{\text {intrinsic }} \\
{[\mathrm{mag}]}\end{array}$ & $\begin{array}{c}T_{\text {eff }} \\
{[\mathrm{K}]}\end{array}$ & $\begin{array}{c}L_{*} \\
{\left[L_{\odot}\right]}\end{array}$ & $\begin{array}{c}\mathcal{M}_{*} \\
{\left[\mathcal{M}_{\odot}\right]}\end{array}$ & $\begin{array}{c}t_{*} \\
{[\mathrm{yr}]}\end{array}$ \\
\hline IRS 9A & $\sim 22$ & $>22000$ & $2.3 \times 10^{5}$ & $\sim 40$ & $\sim 10^{4}$ \\
IRS 9B & $\sim 10$ & $\sim 20000$ & $7.0 \times 10^{3}$ & $\sim 10$ & $\sim 10^{5}$ \\
IRS 9C & $\sim 25$ & $(\sim 20000)$ & $5.5 \times 10^{3}$ & $\sim 9$ & $\sim 10^{5}$ \\
\hline
\end{tabular}

the loci of IRS 9A-C - in particular, at the locus of IRS 9A - are rather insensitive to $T_{\text {eff }}$, one can assume that the mass estimations are barely affected by temperature uncertainties. Certainly, any eventual error in the temperature predominantly affects the age determination. But this setback is somewhat compensated by the fact that the pre-main sequence evolution of high mass stars is extremely rapid anyway, i.e. their loci in the HR diagram shift from the birthline to the zero-age main sequence within a few $10^{4} \mathrm{yr}$. Thus, we consider the above age estimates in the range of $10^{4} \mathrm{yr}$ to $10^{5} \mathrm{yr}$ rather reliable.

Finally we recall: even in the - from the point of our study unlikely case that IRS 9A-C have already arrived on the zeroage main sequence, significant accretion of circumstellar material may still occur, in particular from the circumstellar disk on the central star. The existence of circumstellar disks, seen under different inclinations (e.g. face-on for IRS 9B in contrast to edge-on for IRS 9C), might also provide a reasonable explanation for the relatively large spread of IRS 9A-C's loci in the $(H-K)-(J-H)$ and $(J-K)-M_{J}$ diagrams (Fig. 3). 


\subsection{NGC 3603 IRS 9A-C and the wider environment}

In a recent study we have addressed the question whether the reddened sources IRS 9A-C are related to the NGC 3603 OB cluster (see Fig. 6 of Nürnberger \& Petr-Gotzens 2002). Our deep VLT + ISAAC $K_{\mathrm{s}}$-band imaging data reveal that at the position of IRS 9A-C the radial distribution of stars within the OB cluster does not show any significant excess of star counts. This suggests that IRS 9A-C certainly do not constitute a cluster in its own right, but they might represent a sparse association of protostars, lying well within the boundaries of the NGC 3603 OB cluster.

In addition, we have studied the distribution of dense molecular gas associated with the NGC 3603 starburst region (Nürnberger et al. 2002). As displayed in Fig. 1 and already mentioned in Sect. 1, there is a massive molecular clump (MM 2) located about 1.'3 towards the south of the OB cluster. Following Bohlin et al. (1978) and Dickman (1978) we convert the measured column density $\mathcal{N}\left(\mathrm{H}_{2}\right) \gtrsim 4 \times 10^{22} \mathrm{~cm}^{-2}$ into the extinction of $A_{V} \gtrsim 40^{\mathrm{m}}$ along MM 2 's central line-of-sight. The sources IRS 9A-C are located on the OB cluster facing side of this clump, at the periphery of the gas-free cavity identified by Balick et al. (1980) and Clayton (1986, 1990). By fitting radial profiles to the $\mathrm{C}^{18} \mathrm{O}(2-1)$ emission of the clump and considering the likely $3 \mathrm{D}$ geometry of the whole area we estimate MM 2's contribution to the intrinsic extinction of IRS 9A-C to be at most $A_{V} \sim 4^{\mathrm{m}}$.

Thus, there is strong indication that most of the interstellar material in the vicinity of IRS 9A-C has been dispersed recently by the strong stellar winds originating from the massive stars in the cluster center. We conclude that solely material which is gravitationally bound to the young stellar sources - i.e. gas and dust in their circumstellar envelopes and disks - accounts for the relatively moderate (compared to other protostars) intrinsic extinction values. This would explain why IRS 9A-C are easily detected at near and mid IR wavelengths (which is quite unusual for protostars) while nevertheless they unambiguously show typical signatures of protostars.

\section{Summary and conclusion}

In this paper we have presented new near and mid IR data of the enigmatic, highly reddened sources NGC 3603 IRS 9A-C. Our detailed analysis of the data strongly suggest that IRS 9A-C constitute an association of very young sources of high to intermediate mass. Several age-sensitive parameters, like the slope of the SEDs at IR wavelengths and the source loci in the $(K-N)-(J-K)$ and $(K-N)-M_{K}$ diagrams, indicate that these sources are good candidates for protostars (maybe caught in transition between IR class 0 to IR class I). This last conclusion is supported by IRS 9A's radio fluxes measured with ATCA.

Recently, Moffat et al. (2002) report that IRS 9A itself is not detected in their Chandra ACIS-I data (at a $3 \sigma$ detection level of $4.5 \times 10^{-4} \mathrm{Jy} /$ beam), but they find two nearby X-ray point sources which we consider probably related to neighbouring $K$ band excess sources identified by Tapia et al. (2001). The nondetection of X-ray emission from IRS 9A, however, supports its classification as extremely young source, i.e. protostar.
It is interesting to note that a similar scenario is observed by Beuther et al. (2002) in the deeply embedded star forming region IRAS $19410+2336$. There they detect hard X-ray emission arising from a number of near IR point sources which are most likely intermediate and/or low mass IR class I sources i.e. precursors of Herbig Ae/Be and T Tauri stars, respectively - but they do not detect X-ray emission from the central and most massive source of this cluster.

Anyway, until independent age estimations (e.g. via narrowband near IR imaging and/or spectroscopic data) are derived, the exact evolutionary stage of the sources NGC 3603 IRS 9A-C and the origin of their luminosities (stellar or accretion) will remain open questions. In addition, near/mid IR data with high angular resolution and high sensitivity are essential to investigate the multiplicity of these sources, and will allow to verify whether the fainter $K$ band excess sources (see Tapia et al. 2001) in their vicinity are in the same evolutionary stage.

Acknowledgements. We are indebted to Frank Eisenhauer for providing in electronic format his results from adaptive optics $J H K$ imaging of the NGC 3603 cluster center and to Chris De Pree for kindly providing his ATCA data. Sincere thanks are given to Anita Reimer, nee Mücke, for communicating the $3 \mathrm{~cm}$ and $6 \mathrm{~cm}$ fluxes of IRS 9A prior to publication. It is also a great pleasure to thank our collaborators Leonardo Bronfman, Monika G. Petr-Gotzens, Thomas Stanke, Harold W. Yorke and Hans Zinnecker for their long lasting support of this research project, which has been financed by the Deutsche Forschungsgemeinschaft (DFG) in the framework of its "Physics of Star Formation" program under grants Yo 5/23-1 and Zi 242/22-2. Finally, insightful comments of an anonymous referee are acknowledged.

\section{References}

Adams, F. C., Lada, C. J., \& Shu, F. H. 1987, ApJ, 312, 788 Adams, F. C., Lada, C. J., \& Shu, F. H. 1988, ApJ, 326, 865

André, P., Ward-Thompson, D., \& Barsony, M. 2000, in Protostars and Planets IV, ed. V. Mannings, A. P. Boss, \& S. S. Russell (Tucson: University of Arizona Press), 59

André, P., \& Montmerle, T. 1994, ApJ, 420, 837

Balick, B., Boeshaar, G. O., \& Gull, T. R. 1980, ApJ, 242, 584

Becklin, E. E., Beckwith, S., Gatley, I., et al. 1976, ApJ, 207, 770

Bernasconi, P. A., \& Maeder, A. 1996, A\&A, 307, 829

Bertout, C., Basri, G., \& Bouvier, J. 1988, ApJ, 330, 350

Beuther, H., Kerp, J., Preibisch, Th., Stanke, Th., \& Schilke, P. 2002, A\&A, 395, 169

Bohlin, R. C., Savage, B. D., \& Drake, J. F. 1978, ApJ, 224, 132

Brandl, B., Brandner, W., Eisenhauer, F., et al. 1999, A\&A, 352, L69

Bronfman, L., Nyman, L.-Å., \& May, J. 1996, A\&A, 115, 81

Bronfman, L., May, J., Shepherd, D., \& Nürnberger, D. 2001, in Science with the Atacama Large Millimeter Array, ed. W. Wootten, ASP Conf. Ser., 235, 107

Casali, M. M., \& Hawarden, T. G. 1992, JCMT-UKIRT Newslett., 4, 33

Caswell, J. L. 1998, MNRAS, 297, 215

Caswell, J. L., \& Haynes, R. F. 1987, Aust. J. Phys., 40, 215

Caswell, J. L., Batchelor, R. A., Forster, J. R., \& Wellington, K. J. 1989, Aust. J. Phys., 42, 331

Chini, R. S., Henning, Th., \& Pfau, W. 1991, A\&A, 247, 157

Clayton, C. A. 1986, MNRAS, 219, 895

Clayton, C. A. 1990, MNRAS, 246, 712

Cohen, M. 1998, AJ, 115, 2092 
Crowther, P. A., \& Dessart, L. 1998, MNRAS, 296, 622

D’Antona, F., \& Mazzitelli, I. 1994, ApJS, 90, 467

De Pree, C. G., Nysewander, M. C., \& Goss, W. M. 1999, AJ, 117, 2902

Dickman, R. L. 1978, ApJS, 37, 407

Draine, B. T., \& Lee, H. M. 1984, ApJ, 285, 89

Draine, B. T., \& Li, A. 2001, ApJ, 551, 807

Drissen, L., Moffat, A. F. J., Walborn, N. R., \& Shara, M. M. 1995, AJ, 110, 2235

Ducati, J. R., Bevilacqua, C. M., Rembold, S. B., \& Ribeiro, D. 2001, ApJ, 558, 309

Eisenhauer, F., Quirrenbach, A., Zinnecker, H., \& Genzel, R. 1998, ApJ, 498, 278

Felli, M., \& Stanga, R. 1987, A\&A, 175, 193

Frogel, J. A., Persson, S. E., \& Aaronson, M. 1977, ApJ, 213, 723

Gezari, D. Y., Shu, P., Lamb, G., et al. 1985, ApJ, 299, 1007

Goss, W. M., \& Radhakrishnan, V. 1969, ApL, 4, 199

Haisch, K. E., Lada, E. A., Piña, R. K., Telesco, C. M., \& Lada, C. J. 2001, AJ, 121, 1512

Hildebrand, R. D. 1983, QJRAS, 24, 267

Hofmann, K.-H., Seggewiess, W., \& Weigelt, G. 1995, A\&A, 300, 403

Hog, E., Fabricius, C., Makarov, V. V., et al. 2000, A\&A, 355, L27

Hollenbach, D., Johnston, D., Lizano, S., \& Shu, F. H. 1994, ApJ, 428, 654

Iben, I. 1965, ApJ, 141, 993

Kennicutt, R. C. 1984, ApJ, 287, 116

Koornneef, J. 1983a, A\&AS, 51, 489

Koornneef, J. 1983b, A\&A, 128, 84

Kraemer, K. E., Deutsch, L. K., Jackson, J. M., et al. 1999, ApJ, 516, 817

Krügel, E., \& Siebenmorgen, R. 1994, A\&A, 288, 929

Kurtz, S. E., Watson, A. M., Hofner, P., \& Otte, B. 1999, ApJ, 514, 232

Lada, C. J. 1987, in Star Forming Regions, ed. M. Peimbert, \& J. Jugaku, IAU Symp., 115, 1

Li, A., \& Draine, B. T. 2001, ApJ, 554, 778

Mathis, J. S., Rumpl, W., \& Nordsieck, K. H. 1977, ApJ, 217, 425

Melnick, J., Tapia, M., \& Terlevich, R. 1989, A\&A, 213, 89

Mezger, P. G., \& Henderson, A. P. 1967, ApJ, 147, 471

Moffat, A. F. J. 1983, A\&A, 124, 273

Moffat, A. F. J., Drissen, L., \& Shara, M. M. 1994, ApJ, 436, 183

Moffat, A. F. J., Corcoran, M. F., Stevens, I. R., et al. 2002, ApJ, 573, 191
Mücke, A., Koribalski, B., Moffat, A. F. J., Corcoran, M. F., \& Stevens, I. R. 2002, ApJ, 571, 366

Natta, A., \& Panagia, N. 1976, A\&A, 50, 191

Nielbock, M., Chini, R., Jütte, M., \& Manthey, E. 2001, A\&A, 377, 273

Nürnberger, D. E. A., \& Petr-Gotzens, M. G. 2002, A\&A, 382, 537

Nürnberger, D. E. A., \& Stanke, Th. 2003, A\&A, 400, 223

Nürnberger, D. E. A., Bronfman, L., Yorke, H. W., \& Zinnecker, H. 2002, A\&A, 394, 253

Ossenkopf, V., \& Henning, Th. 1994, A\&A, 291, 943

Pandey, A. K., Ogura, K., \& Sekiguchi, K. 2000, PASJ, 52, 847

Persi, P., Tapia, M., Roth, M., \& Ferrari-Toniolo, M. 1985, A\&A, 144, 275

Persson, S. E., Murphy, D. C., Krzeminski, W., Roth, M., \& Rieke, M. J. 1998, AJ, 116, 2475

Preibisch, Th., Ossenkopf, V., Yorke, H. W., \& Henning, Th. 1993, A\&A, 279, 577

Preibisch, Th., Sonnhalter, C., \& Yorke, H. W. 1995, A\&A, 299, 144

Rieke, G. H., \& Lebofsky, M. J. 1985, ApJ, 288, 618

Roth, M., Tapia, M., Ruiz, M. T., Persi, P., \& Ferrari-Toniolo, M. 1987, in Star Forming Regions, ed. M. Peimbert, \& J. Jugaku, IAU Symp., 115, 182

Rubin, R. H. 1968, ApJ, 154, 391

Sagar, R., Munari, U., \& de Boer, K. S. 2001, MNRAS, 327, 23

Sonnhalter, C., Preibisch, Th., \& Yorke, H. W. 1995, A\&A, 299, 545

Strom, K. M., Strom, S. E., \& Merrill, K. M. 1993, ApJ, 412, 233

Suttner, G., \& Yorke, H. W. 2001, ApJ, 551, 461

Suttner, G., Yorke, H. W., \& Lin, D. N. C. 1999, ApJ, 524, 857

Tapia, M., Bohigas, J., Pérez, B., Roth, M., \& Ruiz, M. T. 2001, RMxAA, 37, 39

Terebey, S., Chandler, C. J., \& André, P. 1993, ApJ, 414, 759

Vacca, W. D., Garmany, C. D., \& Shull, J. M. 1996, ApJ, 460, 914

van der Bliek, N. S., Manfroid, J., \& Bouchet, P. 1996, A\&AS, 119, 547

Weingartner, J. C., \& Draine, B. T. 2001, ApJ, 548, 296

Whittet, D. C. B. 1992, in Dust in the Galactic Environment, The Graduate Series in Astronomy, Institute of Physics Publishing (Bristol)

Yorke, H. W. 1986, ARA\&A, 24, 49

Yorke, H. W., \& Welz, A. 1996, A\&A, 315, 555

Yorke, H. W., \& Sonnhalter, C. 2002, ApJ, 569, 846

Yorke, H. W., Bodenheimer, P., \& Laughlin, G. 1995, ApJ, 443, 199 